J. KSIAM Vol.16, No.1, 15-29, 2012

\title{
A ROBUST AND ACCURATE PHASE-FIELD SIMULATION OF SNOW CRYSTAL GROWTH
}

\author{
YIBAO LI ${ }^{1}$, DONGSUN LEE ${ }^{1}$, HYUN GEUN LEE ${ }^{1}$, DARAE JEONG ${ }^{1}$, CHAEYOUNG LEE ${ }^{1}$, \\ DONGGYU YANG ${ }^{2}$, AND JUNSEOK KIM ${ }^{1 \dagger}$ \\ ${ }^{1}$ Department of Mathematics, Korea University, Seoul 136-701, Republic of Korea \\ E-mail address: cfdkimekorea.ac.kr \\ ${ }^{2}$ Seoul Science High School, Seoul 110-530, Republic of Korea
}

\begin{abstract}
In this paper we introduce 6-fold symmetry crystal growth using new phase-field models based on the modified Allen-Cahn equation. The proposed method is a hybrid method which uses both analytic and numerical solutions. We then show this method can be extended to $k$-fold case. The Wulff construction procedure is provided to understand and predict the shape of crystals. We also present a detailed mathematical proof of the validity of the Wulff construction. For computational results, we verify the accuracy and efficiency of the method for snow crystal growth.
\end{abstract}

\section{INTRODUCTION}

In nonlinear dynamical systems, the physics of phase transformations has attracted considerable interest. Crystal growth is an essential part of phase transformations from the liquid phase to the solid phase via heat transfer. To simulate crystal growth, cellular automaton [25, 44, 45, 46, 47], Monte-Carlo [29, 33], boundary integral [26, 27, 34, 36], front-tracking $[1,12,21]$, level-set $[6,10,17,41]$, and phase-field $[4,5,7,8,11,14,15,16,18,28,30,31$, $32,35,37,39,40,43]$ methods have been developed. Also, many numerical methods such as explicit [12, 13, 16, 31, 39], mixed implicit-explicit [30, 40, 43], and adaptive methods $[5,7,28,32,35]$ have been proposed for crystal growth problems.

Analysis of this paper using the phase field method extends our study to the various cases $k=3,4,5,6, \cdots$, and $n$ with k-fold symmetry. Beside that, one of the crucial to our crystal growth is a method of the multiple time-step algorithm that uses a larger time step for the flowfield calculations while reserving a finer time step for the phase-field evolution was proposed in [37]. Thus, we show that our scheme can give rise to many shapes with $n$-fold symmetry.

In particular, we focus on six-fold symmetric crystal growth, have some physical meaning that snow crystal while, mathematically, we can extend it to the $n$-fold case. In the six-fold case, water has the unique chemical property known as a hydrogen bond. The attractive interaction

Received by the editors August 19 2011; Revised January 30 2012; Accepted in revised form March 52012. 2010 Mathematics Subject Classification. 65M55, 68U10.

Key words and phrases. Allen-Cahn equation, phase-field method, Wulff construction, snow crystal growth.

${ }^{\dagger}$ Corresponding author. 
between the hydrogen and oxygen atoms in different water molecules arranges the solid state water molecules to form a hexagonal shape. For such a reason, in specific temperature, snow crystal grows into six-fold symmetric crystal [24]. The thickness and width of snow crystal are in the ratio of $1: 50$, so snow crystal problems can be simplified into two-dimensional problems. We consider here the solidification of a pure substance from its supercooled melt in two-dimensional space.

In addition, we elaborate on the Wulff construction procedure for the equilibrium crystal shapes with a given interface energy function. We also present a detailed mathematical proof of the validity of the Wulff construction.

This paper is organized as follows: in Section 2, we briefly review basic theoretical concepts about the Wulff construction. The governing equations for crystal growth based on the phasefield medal are given in Section 3. In Section 4, we describe the computationally efficient operator splitting algorithm. In Section 5, we present numerical results of snow crystal growth simulations in 2D. Finally, conclusions are given in Section 6.

\section{THE WULFF CONSTRUCTION}

The equilibrium crystal shape is determined by minimizing the total interfacial free energy. We use the following $k$-fold symmetric interfacial energy equation:

$$
\epsilon(\theta)=\epsilon_{0}\left[1+\epsilon_{k} \cos (k \theta)\right],
$$

where $\epsilon_{0}$ is the mean interfacial tension and $0 \leq \epsilon_{k}<1$ is the anisotropy parameter.

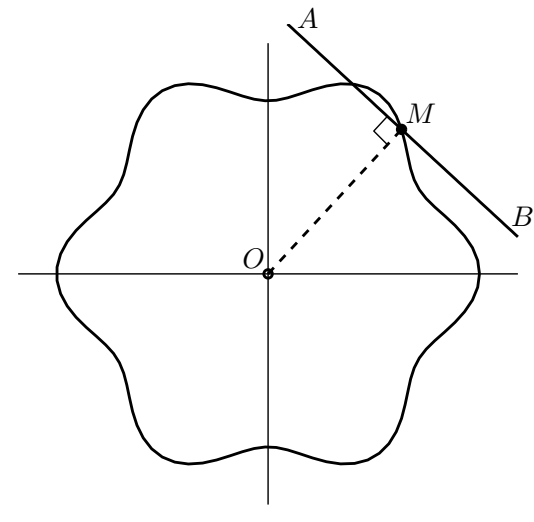

(a)

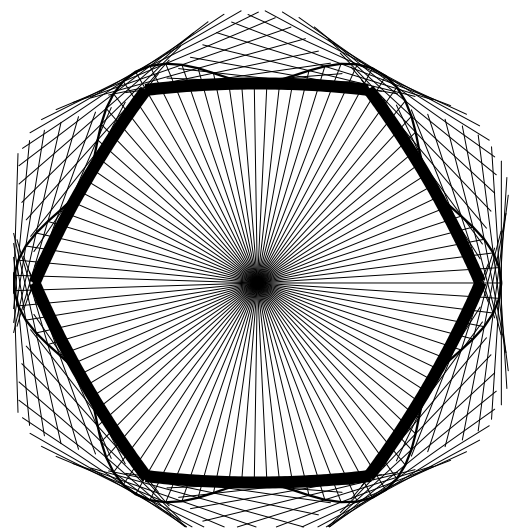

(b)

FIGURE 1. The Wulff construction. (a) Interfacial free-energy density $\epsilon(\theta)$ in the polar coordinates. (b) Equilibrium crystal shape (bold line) for $k=6$, $\epsilon_{0}=1$, and $\epsilon_{6}=0.1$.

In this paper, we focus on $k=6$ case. The equilibrium shape is easily constructed by the Wulff's theorem [42]. We describe the construction of the equilibrium shape geometrically [3]. 
Let $M=(\epsilon(\theta), \theta)$ be a point on the interfacial energy function in the polar coordinates (see Fig. 1(a)). The construction starts from the origin $O$ and draw the line segment $\overline{O M}$ to the point $M$. Draw the perpendicular line $\overleftrightarrow{A B}$ to the line segment $\overline{O M}$. Then the inner convex hull made from all such perpendiculars is an equilibrium crystal shape as shown in Fig. 1(b).

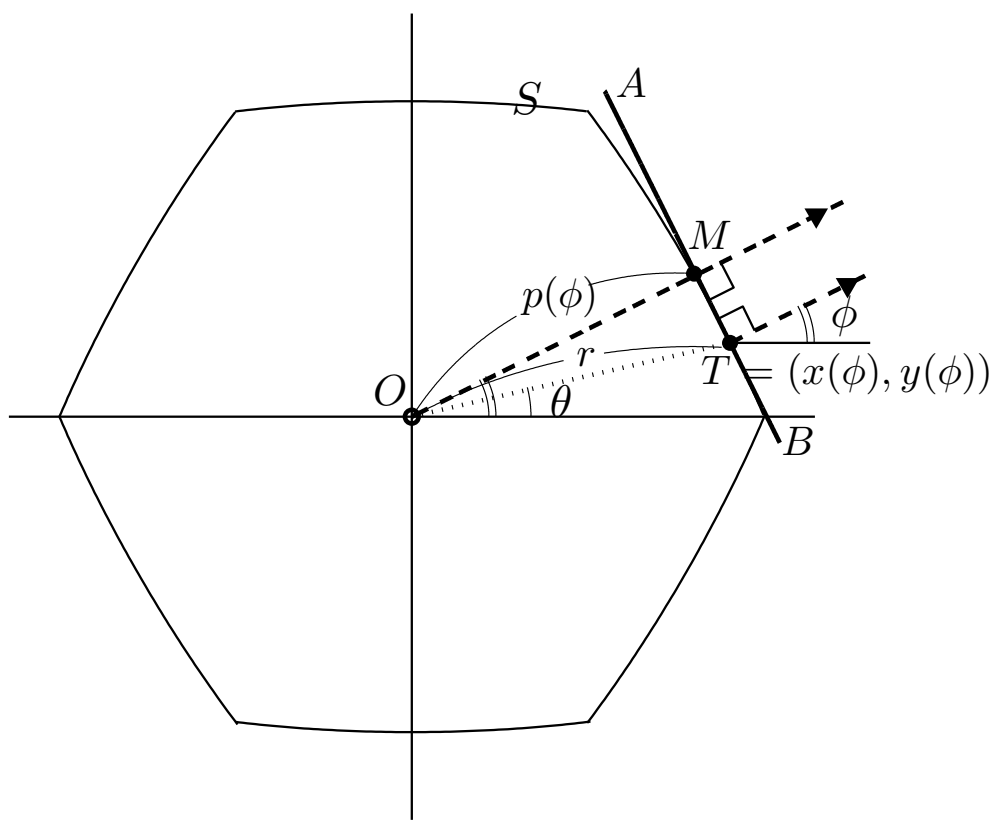

FIGURE 2. Parameter definitions.

Conversely, let us assume the equilibrium shape is known and $(r, \theta)$ be the polar coordinates of a point $T$ of the crystal boundary $S$, that is, $T=(r, \theta)$. And let $T=(x(\phi), y(\phi))$ be the corresponding Cartesian coordinates, where $\phi$ is a parameter and is the angle between $x$-axis and the perpendicular line to the tangent line $\overleftrightarrow{A B}$ at the point $T$. Let $M$ be the intersection point of the line $\overleftrightarrow{A B}$ and the perpendicular line containing the origin to $\overleftrightarrow{A B}$. Let the length of the line segment $\overline{O M}$ be $p(\phi)$. In Fig. 2, we can see these parameter definitions. Then $p(\phi)$ can be obtained from the right triangle $\triangle O T M$ :

$$
p(\phi)=r \cos (\phi-\theta)=r \cos \phi \cos \theta+r \sin \phi \sin \theta=x(\phi) \cos \phi+y(\phi) \sin \phi .
$$

We can express $(x(\phi), y(\phi))$ in terms of $p(\phi)$. First, take a derivative to $p(\phi)$, then we have

$$
p_{\phi}(\phi)=x_{\phi}(\phi) \cos \phi-x(\phi) \sin \phi+y_{\phi}(\phi) \sin \phi+y(\phi) \cos \phi .
$$

Since the normal vector $(\cos \phi, \sin \phi)$ and the tangent vector $\left(x_{\phi}, y_{\phi}\right)$ are orthogonal, that is, $(\cos \phi, \sin \phi) \cdot\left(x_{\phi}, y_{\phi}\right)=0$, we can simplify Eq. (2.2) as

$$
p_{\phi}=-x \sin \phi+y \cos \phi \text {. }
$$


Now, by solving Eqs. (2.1) and (2.3) we have

$$
x(\phi)=p(\phi) \cos \phi-p_{\phi}(\phi) \sin \phi, y(\phi)=p(\phi) \sin \phi+p_{\phi}(\phi) \cos \phi .
$$

Let $F$ and $A$ be the total edge free energy and the area of crystal, respectively and be defined as

$$
\begin{aligned}
F & =\int \epsilon(\phi) \sqrt{\left(x_{\phi}(\phi)\right)^{2}+\left(y_{\phi}(\phi)\right)^{2}} d \phi, \\
A & =\frac{1}{2} \int\left(x(\phi) y_{\phi}(\phi)-y(\phi) x_{\phi}(\phi)\right) d \phi .
\end{aligned}
$$

Using Eq. (2.4), we can rewrite Eqs. (2.5) and (2.6) in the form

$$
\begin{aligned}
F & =\int \epsilon(\phi)\left(p(\phi)+p_{\phi \phi}(\phi)\right) d \phi, \\
A & =\frac{1}{2} \int p(\phi)\left(p(\phi)+p_{\phi \phi}(\phi)\right) d \phi .
\end{aligned}
$$

We want to minimize $F$ with subject to a constant area constraint of $A$. Using the Lagrange multiplier $\lambda$, we seek to minimize

$$
F+\lambda A=\int\left(\epsilon(\phi)+\frac{\lambda}{2} p(\phi)\right)\left(p(\phi)+p_{\phi \phi}(\phi)\right) d \phi .
$$

And then, the Euler-Lagrange equation is

$$
\frac{\partial Q}{\partial p}-\frac{d}{d \phi}\left(\frac{\partial Q}{\partial p_{\phi}}\right)+\frac{d^{2}}{d \phi^{2}}\left(\frac{\partial Q}{\partial p_{\phi \phi}}\right)=0
$$

where

$$
Q=\left(\epsilon+\frac{\lambda}{2} p\right)\left(p+p_{\phi \phi}\right)
$$

From these two Eqs. (2.7) and (2.8), we get

$$
p+p_{\phi \phi}=-\frac{1}{\lambda}\left(\epsilon+\epsilon_{\phi \phi}\right) .
$$

A solution of differential equation (2.9) is

$$
p(\phi)=-\frac{1}{\lambda} \epsilon(\phi)
$$

This result implies that in a crystal at equilibrium, the distances of the faces from the center of the crystal are proportional to their surface free energies per unit area [3].

For large $\epsilon_{6}$ values, the crystal shape will be energy minimizing when certain orientations are missing. Missing orientations occur when the polar plot of $r=1 / \epsilon(\theta)$ changes convexity [9]. The curvature of a polar plot $r(\theta)$ is $\kappa=\left(r^{2}+2 r_{\theta}^{2}-r r_{\theta \theta}\right) /\left(r^{2}+r_{\theta}^{2}\right)^{\frac{3}{2}}$. For $r(\theta)=1 / \epsilon(\theta)$, the curvature is $\kappa=\left(\epsilon+\epsilon_{\theta \theta}\right) /\left[1+\left(\frac{\epsilon_{\theta}}{\epsilon}\right)^{2}\right]^{\frac{3}{2}}$. So convexity changes whenever

$$
\epsilon+\epsilon_{\theta \theta}=\epsilon_{0}\left(1-35 \epsilon_{6} \cos 6 \theta\right)<0 .
$$


If values of $\epsilon_{6}$ are larger than $1 / 35$, then missing orientations occur. In other words, some orientations do not appear on the equilibrium shape of a crystal. Figure 3 shows the 6 -fold Wulff equilibrium shapes $((x(\phi), y(\phi))$ for $0 \leq \phi \leq 2 \pi)$ with two different $\epsilon_{6}$ values: (a) $\epsilon_{6}=1 / 50$ and (b) $\epsilon_{6}=1 / 10$ (which shows the missing orientation). Figure 4 shows the trace of $(x(\phi), y(\phi))$ with different intervals. $\phi_{m}$ is defined as the smallest non-zero value which satisfies $y\left(\phi_{m}\right)=0$.

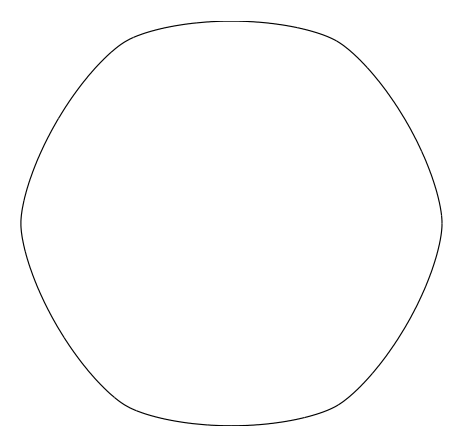

(a) $\epsilon_{6}=1 / 50$

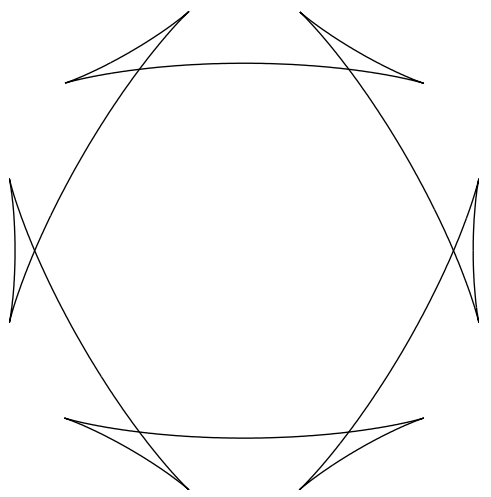

(b) $\epsilon_{6}=1 / 10$

FIGURE 3. The 6-fold Wulff equilibrium shapes with two different $\epsilon_{6}$ values.

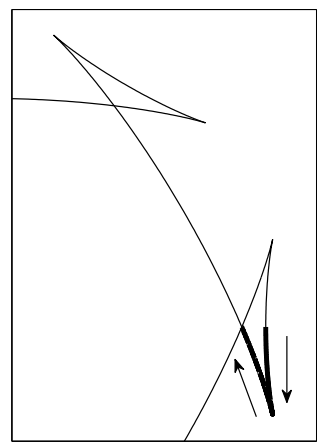

(a) $0 \leq \phi \leq \phi_{m}$

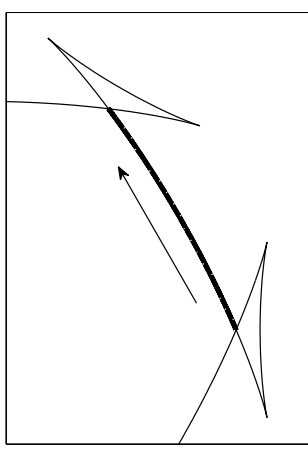

(b) $\phi_{m} \leq \phi \leq \pi / 3-\phi_{m}$

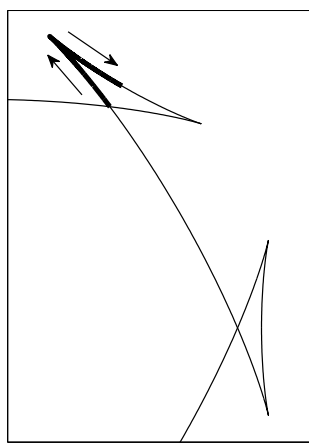

(c) $\pi / 3-\phi_{m} \leq \phi \leq \pi / 3$

FIGURE 4. Trace of $(x(\phi), y(\phi))$ with different intervals and $y\left(\phi_{m}\right)=0$. 


\section{THE PHASE-FIELD MODEL}

The phase-field model for the crystal growth is given by

$$
\begin{aligned}
\epsilon^{2}(c) \frac{\partial c}{\partial t}= & \nabla \cdot\left(\epsilon^{2}(c) \nabla c\right)+\left[c-\lambda U\left(1-c^{2}\right)\right]\left(1-c^{2}\right) \\
& +\left(|\nabla c|^{2} \epsilon(c) \frac{\partial \epsilon(c)}{\partial c_{x}}\right)_{x}+\left(|\nabla c|^{2} \epsilon(c) \frac{\partial \epsilon(c)}{\partial c_{y}}\right)_{y} \\
\frac{\partial U}{\partial t}= & D \Delta U+\frac{1}{2} \frac{\partial c}{\partial t},
\end{aligned}
$$

where $c$ is the order parameter, $\epsilon(c)$ is the anisotropic function, $\lambda$ is the dimensionless coupling parameter, and $U=c_{p}\left(T-T_{M}\right) / L$ is the dimensionless temperature field. Here $c_{p}$ is the specific heat at constant pressure, $T_{M}$ is the melting temperature, $L$ is the latent heat of fusion, $D=\alpha \tau_{0} / \epsilon_{0}^{2}, \alpha$ is the thermal diffusivity, $\tau_{0}$ is the characteristic time, and $\epsilon_{0}$ is the characteristic length. The order parameter is defined by $c=1$ in the solid phase and $c=-1$ in the liquid phase. The interface is defined by $c=0$ and $\lambda$ is given as $\lambda=D / a_{2}$ with $a_{2}=0.6267$ $[15,16]$. We define a normal vector of $c$ as $\left(c_{x}, c_{y}\right)$ and an angle between normal vector and $x$ axis as $\phi$ that satisfies $\tan \phi=c_{y} / c_{x}$. Then by replacing $\epsilon(c)$ with $\epsilon(\phi)=\epsilon_{0}\left(1+\epsilon_{6} \cos (6 \phi)\right)$, we can simplify the following terms in Eq. (3.1):

$$
\left(|\nabla c|^{2} \epsilon(\phi) \frac{\partial \epsilon(\phi)}{\partial c_{x}}\right)_{x}=\left(\left(c_{x}^{2}+c_{y}^{2}\right) \epsilon(\phi) \epsilon^{\prime}(\phi)\left(-\frac{c_{y}}{c_{x}^{2}+c_{y}^{2}}\right)\right)_{x}=-\left(\epsilon^{\prime}(\phi) \epsilon(\phi) c_{y}\right)_{x} .
$$

In a similar way, we get

$$
\left(|\nabla c|^{2} \epsilon(\phi) \frac{\partial \epsilon(\phi)}{\partial c_{y}}\right)_{y}=\left(\epsilon^{\prime}(\phi) \epsilon(\phi) c_{x}\right)_{y} .
$$

Hence we can rewrite the governing equations of 6-fold symmetric crystal growth as following:

$$
\begin{aligned}
\epsilon^{2}(\phi) \frac{\partial c}{\partial t}= & \nabla \cdot\left(\epsilon^{2}(\phi) \nabla c\right)+\left[c-\lambda U\left(1-c^{2}\right)\right]\left(1-c^{2}\right) \\
& -\left(\epsilon^{\prime}(\phi) \epsilon(\phi) c_{y}\right)_{x}+\left(\epsilon^{\prime}(\phi) \epsilon(\phi) c_{x}\right)_{y} \\
\frac{\partial U}{\partial t}= & D \Delta U+\frac{1}{2} \frac{\partial c}{\partial t} .
\end{aligned}
$$

\section{NUMERICAL SOLUTION}

In this section, we propose a robust hybrid numerical method for crystal growth simulation. For simplicity of exposition we shall discretize Eqs. (3.2) and (3.3) in two-dimensional space, i.e., $\Omega=\left(-l_{1}, l_{1}\right) \times\left(-l_{2}, l_{2}\right)$. Let $N_{x}$ and $N_{y}$ be positive even integers, $h=2 l_{1} / N_{x}$ be the uniform mesh size, and $\Omega_{h}=\left\{\left(x_{i}, y_{j}\right): x_{i}=(i-0.5) h, y_{j}=(j-0.5) h, 1 \leq i \leq\right.$ $\left.N_{x}, 1 \leq j \leq N_{y}\right\}$ be the set of cell-centers. Let $c_{i j}^{n}$ be approximations of $c\left(x_{i}, y_{j}, n \Delta t\right)$, where $\Delta t=T / N_{t}$ is the time step, $T$ is the final time, and $N_{t}$ is the total number of time steps. The discrete differentiation operator is $\nabla_{d} c_{i j}=\left(c_{i+1, j}-c_{i-1, j}, c_{i, j+1}-c_{i, j-1}\right) /(2 h)$. We 
then define the discrete Laplacian by $\Delta_{d} c_{i j}=\left(c_{i+1, j}+c_{i-1, j}-4 c_{i j}+c_{i, j+1}+c_{i, j-1}\right) / h^{2}$. We discretize Eqs. (3.2) and (3.3):

$$
\begin{aligned}
\epsilon^{2}\left(\phi^{n}\right) \frac{c^{n+1}-c^{n}}{\Delta t}= & \epsilon^{2}\left(\phi^{n}\right) \Delta_{d} c^{n+1,2}+2 \epsilon\left(\phi^{n}\right) \nabla_{d} \epsilon\left(\phi^{n}\right) \cdot \nabla_{d} c^{n} \\
& -F^{\prime}\left(c^{n+1}\right)-4 \lambda U^{n} F\left(c^{n+1,1}\right) \\
& -\left(\epsilon^{\prime}(\phi) \cdot \epsilon(\phi) c_{y}\right)_{x}^{n}+\left(\epsilon^{\prime}(\phi) \cdot \epsilon(\phi) c_{x}\right)_{y}^{n}, \\
\frac{U^{n+1}-U^{n}}{\Delta t}= & D \Delta_{d} U^{n+1}+\frac{c^{n+1}-c^{n}}{2 \Delta t},
\end{aligned}
$$

where $F(c)=0.25\left(c^{2}-1\right)^{2}$ and $F^{\prime}(c)=c\left(c^{2}-1\right)$. Here $c^{n+1, k}$ for $k=1,2$ are defined in the operator splitting scheme. We propose the following operator splitting scheme:

$$
\begin{aligned}
\epsilon^{2}\left(\phi^{n}\right) \frac{c^{n+1,1}-c^{n}}{\Delta t}= & 2 \epsilon\left(\phi^{n}\right) \nabla_{d} \epsilon\left(\phi^{n}\right) \cdot \nabla_{d} c^{n} \\
& -\left(\epsilon^{\prime}(\phi) \cdot \epsilon(\phi) c_{y}\right)_{x}^{n}+\left(\epsilon^{\prime}(\phi) \cdot \epsilon(\phi) c_{x}\right)_{y}^{n}, \\
\epsilon^{2}\left(\phi^{n}\right) \frac{c^{n+1,2}-c^{n+1,1}}{\Delta t}= & \epsilon^{2}\left(\phi^{n}\right) \Delta_{d} c^{n+1,2}-4 \lambda U^{n} F\left(c^{n+1,1}\right), \\
\epsilon^{2}\left(\phi^{n}\right) \frac{c^{n+1}-c^{n+1,2}}{\Delta t}= & -F^{\prime}\left(c^{n+1}\right) .
\end{aligned}
$$

We can solve Eq. (4.1) analytically by the method of separation of variables [22, 23]. The solution is given as follows:

$$
c^{n+1}=\frac{c^{n+1,2}}{\sqrt{e^{-\frac{2 \Delta t}{\epsilon^{2}\left(\phi^{n}\right)}}+\left(c^{n+1,2}\right)^{2}\left(1-e^{-\frac{2 \Delta t}{\epsilon^{2}\left(\phi^{n}\right)}}\right)}} .
$$

Finally, the proposed scheme can be written as follows:

$$
\begin{aligned}
& \epsilon\left(\phi^{n}\right) \frac{c^{n+1,1}-c^{n}}{\Delta t}=2 \epsilon\left(\phi^{n}\right)_{x} c_{x}^{n}+2 \epsilon\left(\phi^{n}\right)_{y} c_{y}^{n}-\left(\epsilon^{\prime}(\phi) \cdot c_{y}\right)_{x}^{n}+\left(\epsilon^{\prime}(\phi) \cdot c_{x}\right)_{y}^{n}, \\
& \epsilon^{2}\left(\phi^{n}\right) \frac{c^{n+1,2}-c^{n+1,1}}{\Delta t}=\epsilon^{2}\left(\phi^{n}\right) \Delta_{d} c^{n+1,2}-4 \lambda U^{n} F\left(c^{n+1,1}\right), \\
& c^{n+1}=\frac{c^{n+1,2}}{\sqrt{e^{-\frac{2 \Delta t}{\epsilon^{2}\left(\phi^{n}\right)}}+\left(c^{n+1,2}\right)^{2}\left(1-e^{-\frac{2 \Delta t}{\epsilon^{2}\left(\phi^{n}\right)}}\right)}}, \\
& \frac{U^{n+1}-U^{n}}{\Delta t}=D \Delta_{d} U^{n+1}+\frac{c^{n+1}-c^{n}}{2 \Delta t} .
\end{aligned}
$$

Equations (4.2) and (4.3) can be solved by a multigrid method [2, 38]. 


\section{NUMERICAL RESULTS}

In this section we perform numerical experiments for two-dimensional solidification to validate that our proposed scheme is accurate, efficient, and robust. Unless otherwise specified, we take the initial state as

$$
c(x, y, 0)=\tanh \left(\frac{R_{0}-\sqrt{x^{2}+y^{2}}}{\sqrt{2}}\right) \text { and } U(x, y, 0)= \begin{cases}0 & \text { if } c>0 \\ \Delta & \text { else. }\end{cases}
$$

The zero level set $(c=0)$ represents a circle of radius $R_{0}$. From the dimensionless variable definition the value $U=0$ corresponds to the melting temperature of the pure material, while $U=\Delta$ is the initial undercooling. The capillary length, $d_{0}$, is defined as $d_{0}=a_{1} / \lambda[4,20,32]$ with $a_{1}=0.8839[15,16,32]$ and $\lambda=3.1913$ [32].

5.1. Convergence test. To obtain an estimate of the convergence rate, we perform a number of simulations for 6 -fold crystal growth problem on a set of increasingly finer grids. The computational domain is $\Omega=(-100,100)^{2}$ and we take $R_{0}=15 d_{0}, \epsilon_{6}=0.02$, and $\Delta=$ -0.55 . The numerical solutions are computed on the uniform grids $h=200 / 2^{n}$ and with corresponding time steps $\Delta t=0.6 / 2^{n-8}$ for $n=8,9,10$, and 11 . The calculations are run up to time $T=150$. We define the error to be the discrete of $l_{2}$-norm of the difference between that grid and the average of the next finer grid cells covering it:

$$
e_{h / \frac{h}{2}}{ }_{i j}=c_{h i j}-\left(c_{\frac{h}{2} 2 i-1,2 j-1}+c_{\frac{h}{2} 2 i-1,2 j}+c_{\frac{h}{2} 2 i, 2 j-1}+c_{\frac{h}{2} 2 i, 2 j}\right) / 4 .
$$

The rate of convergence is defined as:

$$
\log _{2}\left(\left\|e_{h / \frac{h}{2}}\right\|_{2} /\left\|e_{\frac{h}{2} / \frac{h}{4}}\right\|_{2}\right) .
$$

The errors and rates of convergence are given in Table 1 . The results suggest that the scheme is indeed second order accurate in space. Figure 5 shows the convergence of numerical results under mesh refinement.

TABLE 1. Error and $l_{2}$ convergence result.

\begin{tabular}{|c|c|c|c|c|}
\hline $256-512$ & Rate & $512-1024$ & Rate & $1024-2048$ \\
\hline $5.477 \mathrm{E}-4$ & 1.96 & $1.405 \mathrm{E}-4$ & 2.01 & $3.487 \mathrm{E}-5$ \\
\hline
\end{tabular}

Next, we consider the evolution of the interface with different time steps in order to investigate the effect of time step. A $1024 \times 1024$ mesh is used on the domain $\Omega=(-200,200)^{2}$ with $R_{0}=50 d_{0}, \epsilon_{6}=0.02$, and $\Delta=-0.55$. Figure 6(a) shows the interfaces at time $T=1200$ with different time steps $\Delta t=0.6,0.3$, and 0.15 . Figure $6(\mathrm{~b})$ shows the velocity of the tip versus time. For the calculation of the crystal tip velocity, refer to Ref. [22]. The velocity $V$ of the tip at time $T=1200$ versus time step is shown in Fig. 6(c). Here, we define the error between the fitting velocity $\widetilde{V}$ and $V$ as $E_{i}=\left|\widetilde{V}_{i}-V_{i}\right| / V_{i}$. In Fig. 6(c), the linear fit $\widetilde{V}$ is done using the MATLAB function "polyfit" and the errors on the index $i$ are calculated by the MATLAB function "polyval" on the results of the linear fit. In this test, the $l_{2}$ error is $0.54 \%$. 


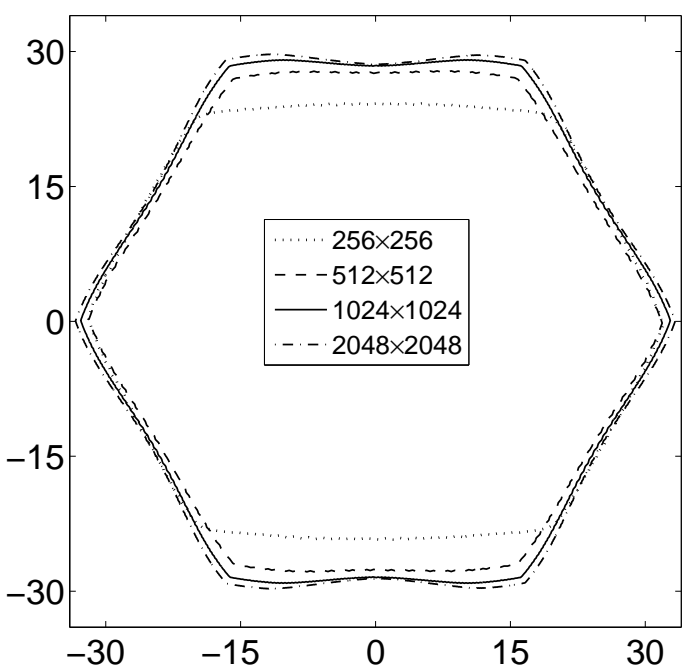

FIGURE 5. Convergence of numerical results under mesh refinement.

Therefore the results suggest that the convergence rate of the tip velocity is linear with respect to the time step.

5.2. Stability test. In this section, we perform a number of simulations on a set of increasingly finer grids to show that our proposed method is more stable than the previous methods which suffer from time restrictions $\Delta t \leq O\left(h^{2}\right)$ for stability. The computational domain is $\Omega=$ $(-200,200)^{2}$ and we take $R_{0}=15 d_{0}, \epsilon_{6}=0.02$, and $\Delta=-0.55$. The numerical solutions are computed on the uniform grids $h=400 / 2^{n}$ with corresponding time steps $\Delta t=3 h$ for $n=8,9$, and 10. Figure 7 shows the crystal growth with different time steps at $T=70.31$. In general, large time steps may cause large truncation errors. However, as can be seen in Fig. 7, we obtain stable solutions with large time steps.

Next, we calculate the maximum $\Delta t$ corresponding to different spatial grid sizes $h$ so that stable solutions can be computed after 20 time step iterations. The results are shown in Table 2 and we obtain stable solutions for all three mesh sizes. Note that there is a linear relation between the time step and mesh sizes. Thus, for finer mesh sizes we may use larger time steps than previous conventional methods.

TABLE 2. Stability constraint of $\Delta t$ for the proposed scheme.

\begin{tabular}{|c|c|c|c|}
\hline Mesh size & $h=400 / 256$ & $h=400 / 512$ & $h=400 / 1024$ \\
\hline Time step & $\Delta t \leq 12 h$ & $\Delta t \leq 10 h$ & $\Delta t \leq 8 h$ \\
\hline
\end{tabular}

5.3. Effect of $\epsilon_{6}$. To investigate the effect of $\epsilon_{6}$, we consider the evolution of the interface with different $\epsilon_{6}=0.002,0.02$, and 0.05 . A $1024 \times 1024$ mesh is used on the domain $\Omega=$ 


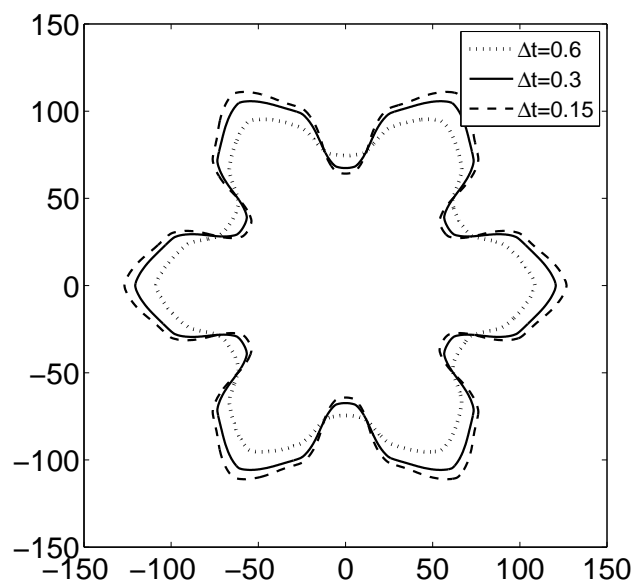

(a)

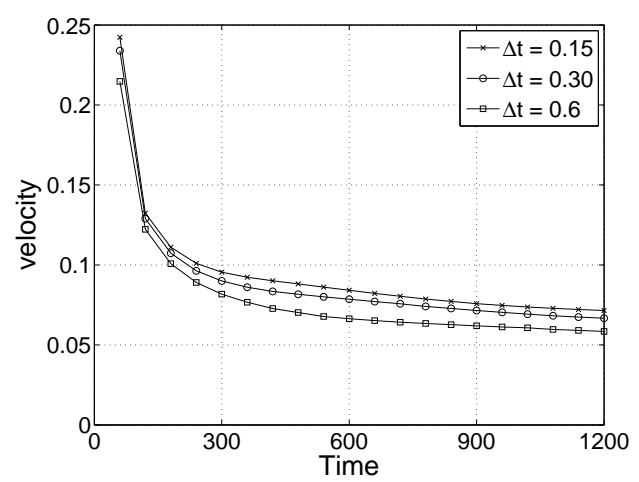

(b)

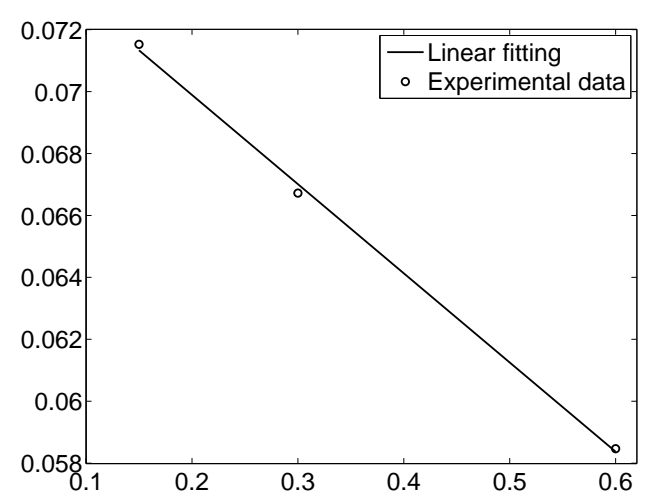

(c)

FIGURE 6. (a) The interfaces at $T=1200$ for different time steps. (b) shows the velocity of the tip versus time. (c) The numerical experimental and linear fitting velocities versus time step.

$(-100,100)^{2}$ and we take $R_{0}=50 d_{0}, \Delta=-0.55, \Delta t=0.3$, and $T=1200$. Figures 8(a), (b), and (c) are the evolution of crystal growth with $\epsilon_{6}=0.002,0.02$, and 0.05 , respectively. As advised in the previous paper, If $\epsilon_{6}<\frac{1}{35}$, all of tangent planes lie outside and all orientations appear on the equilibrium shape. Detail view is drawn in Fig. 8(a). Otherwise, there is missing orientations shown in Fig. 8(c). While if $\epsilon_{6}$ is not more smaller than $\frac{1}{35}$, the crystal also works well shown in Fig. 8(b). Thus the Wulff construction is not strictly correlated with $\epsilon_{6}$ in crystal growth, but provide guidelines for parameter selection.

5.4. Effect of undercooling. Now we investigate the effects of undercooling of the initial solid seed. For each test, a $1024 \times 1024$ mesh is used on the domain $\Omega=(-200,200)^{2}$ and we choose $R_{0}=15 d_{0}, \epsilon_{6}=0.02, \Delta t=0.3$, and $T=1080$. Figure 9 shows sequences of 

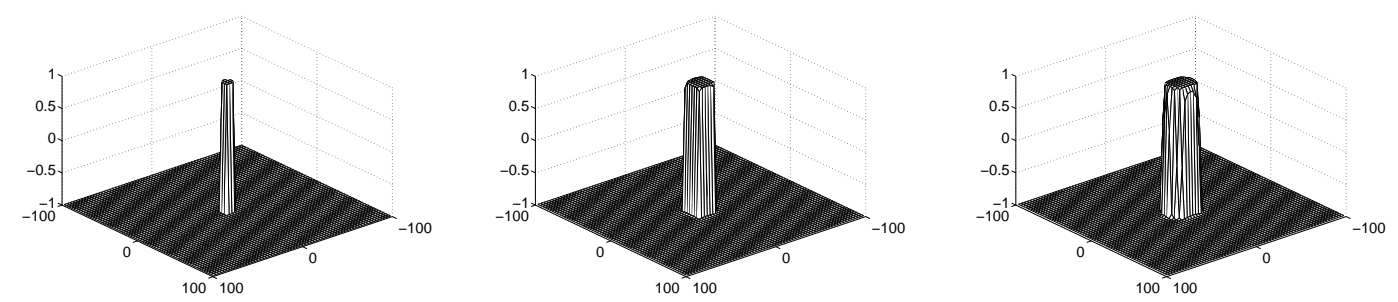

FIGURE 7. The stability of crystal growth with different mesh sizes: (a) $256 \times$ 256 mesh $(\Delta t=4.68)$, (b) $512 \times 512$ mesh $(\Delta t=2.34)$, and (c) $1024 \times 1024$ $\operatorname{mesh}(\Delta t=1.17)$.

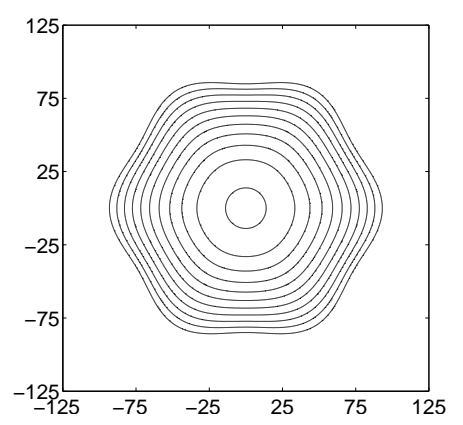

(a)

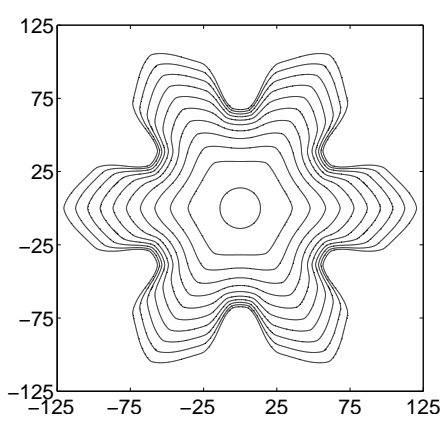

(b)

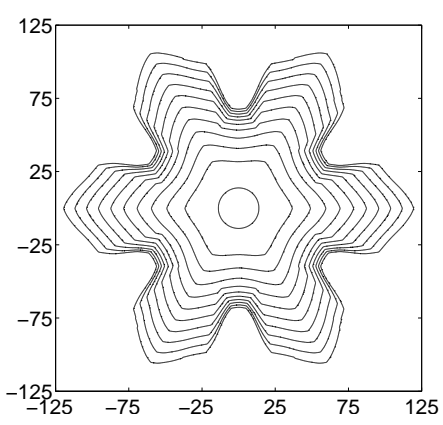

(c)

FIGURE 8. The effect of $\epsilon_{6}$. (a), (b), and (c) are the evolution of crystal growth with $\epsilon_{6}=0.002,0.02$, and 0.05 , respectively. The times are $t=0,120,240$, $360,480,600,720,840,960,1080$, and 1200 .

interfaces with different undercooling sizes $\Delta=-0.45, \Delta=-0.55$, and $\Delta=-0.65$. We observe that the large initial undercooling causes the dendrite to grow faster.

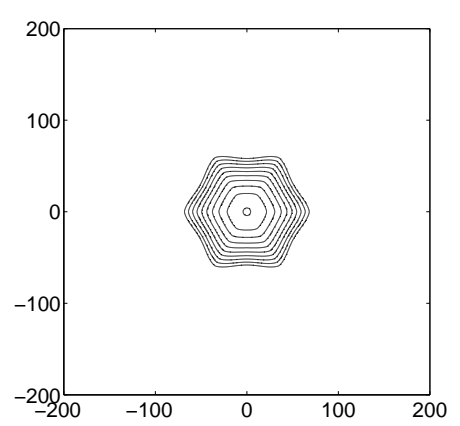

(a) $\Delta=-0.45$

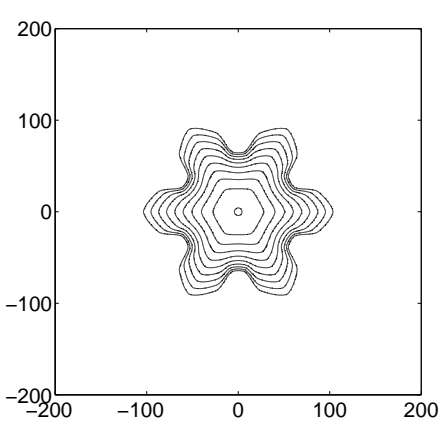

(b) $\Delta=-0.55$

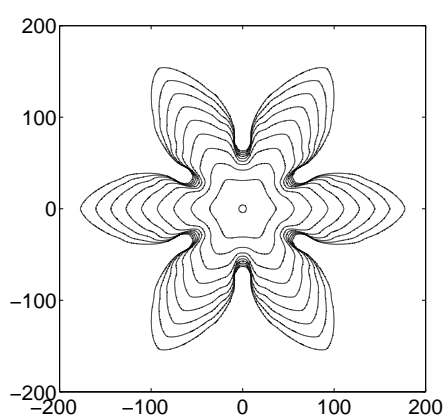

(c) $\Delta=-0.65$

FIGURE 9. Sequences of interfaces with different undercooling sizes $\Delta=$ $-0.45, \Delta=-0.55$, and $\Delta=-0.65$. 
5.5. $k$-fold symmetric crystal growth. If we set the energy function by $\epsilon(\phi)=\epsilon_{0}(1+$ $\left.\epsilon_{k} \cos (k \phi)\right)$, then our proposed method can simulate the $k$-fold crystal growth in general. To show this, we simulate sequences of computational experiments of $k$-fold symmetric crystal growth for $k=4, \ldots, 9$. A $1024 \times 1024$ mesh is used on the domain $\Omega=(-200,-200)^{2}$ and we take $R_{0}=15 d_{0}, \Delta=-0.55$, and $\Delta t=0.3$. Note that we use $\epsilon_{k}=1 /\left(k^{2}-1\right)$ to respond to the Wulff's algorithm. The evolutions for each $k$ are shown in Fig. 10.

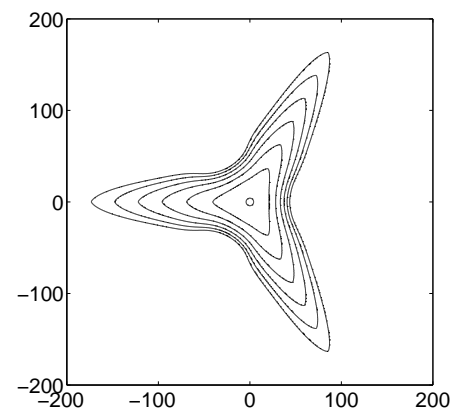

(a) $k=3$

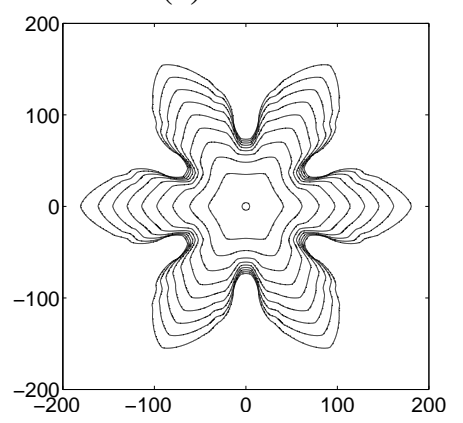

(d) $k=6$

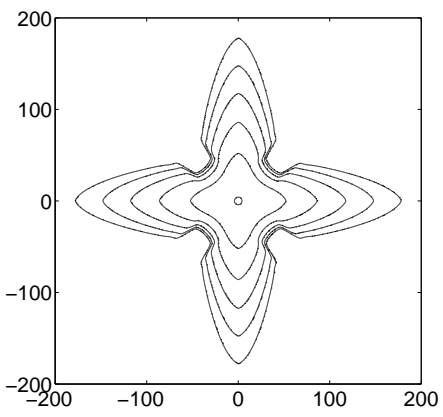

(b) $k=4$

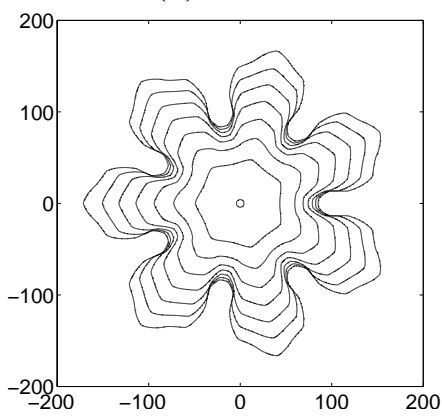

(e) $k=7$

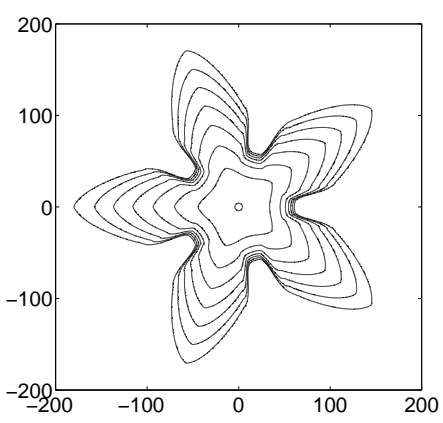

(c) $k=5$

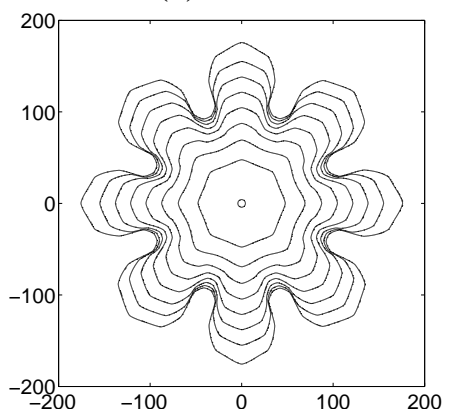

(f) $k=8$

FIGURE 10. The evolutions of $k$-fold crystal growth after time: (a) $T=720$, (b) $T=1200$, (c) $T=1680$, (d) $T=2160$, (e) $T=2520$, and (f) $T=2880$.

5.6. Comparison with the previous study. An isotropic finite-difference scheme for simulating 6 -fold symmetric dendritic solidification is presented in [19]. The author showed that the stability criterion becomes $\Delta t \leq(3 / 8) h^{2}$. But, as we can see in Section 5.2, the time restriction of our proposed method is $\Delta t \sim O(h)$. In order to show the improvement of our proposed method, we use the same numerical parameters as in [19], e.g., $\lambda=1.7680, \epsilon_{0}=1.1312$, $\epsilon_{6}=0.05, D=2$, and $R_{0}=5$. Note that in [19], the author took the step size as $h=0.4$ in the progressively increased mesh sizes as $500 \times 500$ for $0 \leq t \leq 150$, to $800 \times 800$ for $150 \leq t \leq 250$, and to $1200 \times 1200$ for $250 \leq t \leq 400$. Here we take a $1280 \times 1280$ mesh size. This simulation is run up to $T=400$ with $\Delta t=0.2$. Our proposed method took about only 5 hours of CPU time, which is drastically reduced faster than the CPU time (1000 hours) in [19]. 


\section{CONCLUSION}

In this paper we presented an accurate and efficient numerical method for phase-field models of $k$-fold snow crystal growth. We described the Wulff construction procedure for the equilibrium crystal shapes with a given interface energy function. For the interfacial energy larger than a particular value, convexity changes and missing orientations occur. Focusing on 6 -fold symmetric shape, we calculated the particular value. We also provided a detailed mathematical proof of the validity of the Wulff construction. The proposed method is a hybrid method which uses both analytic and numerical solutions. We extended the model to $k$-fold symmetric crystal growth. Computational results showed the accuracy and efficiency of the method.

\section{ACKNOWLEDGMENTS}

This work was supported by Seoul Science High School R\&E program in 2011. The authors wish to thank the reviewers for the constructive and helpful comments on the revision of this article.

\section{REFERENCES}

[1] N. Al-Rawahi and G. Tryggvason, Numerical simulation of dendritic solidification with convection: twodimensional geometry, Journal of Computational Physics, 180 (2002), 471-496.

[2] W.L. Briggs, A Multigrid Tutorial, SIAM, Philadelphia, 1987.

[3] W.K. Burton, N. Cabrera, and F.C. Frank, The growth of crystals and the equilibrium structure of their surfaces, Philosophical Transactions of the Royal Society A, 243 (1951), 299-358.

[4] G. Caginalp, Stefan and Hele-Shaw type models as asymptotic limits of the phase-field equations, Physical Review A, 39 (1989), 5887-5896.

[5] C.C. Chen and C.W. Lan, Efficient adaptive three-dimensional phase-field simulation of dendritic crystal growth from various supercoolings using rescaling, Journal of Crystal Growth, 311 (2009), 702-706.

[6] S. Chen, B. Merriman, S. Osher, and P. Smereka, A simple level set method for solving Stefan problem, Journal of Computational Physics, 135 (1997), 8-29.

[7] C.C. Chen, Y.L. Tsai, and C.W. Lan, Adaptive phase field simulation of dendritic crystal growth in a forced flow: $2 D$ vs. $3 D$ morphologies, International Journal of Heat and Mass Transfer, 52 (2009), 1158-1166.

[8] J.-M. Debierre, A. Karma, F. Celestini, and R. Guérin, Phase-field approach for faceted solidification, Physical Review E, 68 (2003), 041604.

[9] F.C. Frank, Metal Surfaces, ASM, Cleveland, OH, 1963.

[10] F. Gibou, R. Fedkiw, R. Caflisch, and S. Osher, A level set approach for the numerical simulation of dendritic growth, Journal of Scientific Computing, 19 (2002) 183-199.

[11] J.-H. Jeong, N. Goldenfeld, and J.A. Dantzig, Phase field model for three-dimensional dendritic growth with fluid flow, Physical Review E, 64 (2001), 041602.

[12] D. Juric and G. Tryggvason, A front-tracking method for dendritic solidification, Journal of Computational Physics, 123 (1996), 127-148.

[13] A. Jacot and M. Rappaz, A pseudo-front tracking technique for the modelling of solidification microstructures in multi-component alloys, Acta Materialia, 50 (2002), 1909-1926.

[14] A. Karma, Y.H. Lee, and M. Plapp, Three-dimensional dendrite-tip morphology at low undercooling, Physical Review E, 61 (2000) 3996-4006.

[15] A. Karma and W.-J. Rappel, Phase-field method for computationally efficient modeling of solidification with arbitrary interface kinetics, Physical Review E, 53 (1996), 3017-3020. 
[16] A. Karma and W.-J. Rappel, Quantitative phase-field modeling of dendritic growth in two and three dimensions, Physical Review E, 57 (1998), 4323-4349.

[17] Y.-T. Kim, N. Goldenfeld, and J. Dantzig, Computation of dendritic microstructures using a level set method, Physical Review E, 62 (2000) 2471-2474.

[18] R. Kobayashi, Modeling and numerical simulations of dendritic crystal growth, Physica D, 63 (1993), 410423.

[19] A. Kumar, Isotropic finite-differences, Journal of Computational Physics, 201 (2004), 109-118.

[20] J.S. Langer, Directions in Condensed Matter, World Scientific, Singapore, 1986, 164-186.

[21] X. Li, J. Glimm, X. Jiao, C. Peyser, and Y. Zhao, Study of crystal growth and solute precipitation through front tracking method, Acta Mathematica Scientia, 30 (2010), 377-390.

[22] Y. Li, H.G. Lee, and J.S. Kim, A fast, robust, and accurate operator splitting method for phase-field simulations of crystal growth, Journal of Crystal Growth, 321 (2011), 176-182.

[23] Y. Li, H.G. Lee, D. Jeong, and J.S. Kim, An unconditionally stable hybrid numerical method for solving the Allen-Cahn equation, Computers and Mathematics with Applications, 60 (2010), 1591-1606.

[24] K.G. Libberecht, The physics of snow crystal, Reports on Progress in Physics, 68 (2005), 855-895.

[25] D. Li, R. Li, and P. Zhang, A cellular automaton technique for modelling of a binary dendritic growth with convection, Applied Mathematical Modelling, 31 (2007), 971-982.

[26] S. Li, J.S. Lowengrub, P.H. Leo, and V. Cristini, Nonlinear stability analysis of self-similar crystal growth: control of the Mullins-Sekerka instability, Journal of Crystal Growth, 277 (2005), 578-592.

[27] D.I. Meiron, Boundary integral formulation of the two-dimensional symmetric model of dendritic growth, Physica D, 23 (1986), 329-339.

[28] N. Provatas, N. Goldenfeld, and J. Dantzig, Efficient computation of dendritic microstructures using adaptive mesh refinement, Physical Review Letters, 80 (1998), 3308-3311.

[29] M. Plapp and A. Karma, Multiscale finite-difference-diffusion-Monte-Carlo method for simulating dendritic solidification, Journal of Computational Physics, 165 (2000), 592-619.

[30] N. Provatas, N. Goldenfeld, and J. Dantzig, Adaptive mesh refinement computation of solidification microstructures using dynamic data structures, Journal of Computational Physics, 148 (1999), 265-290.

[31] J.C. Ramirez, C. Beckermann, A. Karma, and H.-J. Diepers, Phase-field modeling of binary alloy solidification with coupled heat and solute diffusion, Physical Review E, 69 (2004), 051607.

[32] J. Rosam, P.K. Jimack, A. Mullis, A fully implicit, fully adaptive time and space discretisation method for phase-field simulation of binary alloy solidification, Journal of Computational Physics, 225 (2007), 12711287.

[33] T.P. Schulze, Simulation of dendritic growth into an undercooled melt using kinetic Monte Carlo techniques, Physical Review E, 78 (2008), 020601.

[34] J.A. Sethian and J. Straint, Crystal growth and dendritic solidification, Journal of Computational Physics, 98 (1992), 231-253.

[35] C.J. Shih, M.H. Lee, and C.W. Lan, A simple approach toward quantitative phase field simulation for dilutealloy solidification, Journal of Crystal Growth, 282 (2005), 515-524.

[36] J. Strain, A boundary integral approach to unstable solidification, Journal of Computational Physics, 85 (1989), 342-389.

[37] X. Tong, C. Beckermann, A. Karma, and Q. Li, Phase-field simulations of dendritic crystal growth in a forced flow, Physical Review E, 63 (2001), 061601.

[38] U. Trottenberg, C. Oosterlee, and A. Schüller, Multigrid, Academic Press, USA, 2001.

[39] J.A. Warren and W.J. Boettinger, Prediction of dendritic growth and microsegregation patterns in a binary alloy using the phase-field method, Acta Metallurgica et Materialia, 43 (1995), 689-703.

[40] S.-L. Wang and R.F. Sekerka, Algorithms for phase field computation of the dendritic operating state at large supercoolings, Journal of Computational Physics, 127 (1996), 110-117.

[41] K. Wang, A. Chang, L.V. Kale, and J.A. Dantzig, Parallelization of a level set method for simulating dendritic growth, Journal of Parallel and Distributed Computing, 66, (2006), 1379-1386. 
[42] G. Wulff, Zur frage der geschwindigkeit des wachsturms under auflosung der kristallfachen, Z Kristallogr, 34 (1901), 449-530.

[43] Y. Xu, J.M. McDonough and K.A. Tagavi, A numerical procedure for solving $2 D$ phase-field model problems, Journal of Computational Physics, 218 (2006), 770-793.

[44] H. Yin and S.D. Felicelli, A cellular automaton model for dendrite growth in magnesium alloy AZ91, Modelling Simul, Materials Science and Engineering, 17 (2009), 075011.

[45] M.F. Zhu and C.P. Hong, A modified cellular automaton model for the simulation of dendritic growth in solidification of alloys, ISIJ International, 41 (2001), 436-445.

[46] M.F. Zhu, S.Y. Lee, and C.P. Hong, Modified cellular automaton model for the prediction of dendritic growth with melt convection, Physical Review E, 69 (2004), 061610.

[47] M.F. Zhu, S.Y. Pan, D.K. Sun, and H.L. Zhao, Numerical simulation of microstructure evolution during alloy solidification by using cellular automaton method, ISIJ International, 50 (2010), 1851-1858. 